\title{
E+A Galaxies: Did They Lose The A to Become E?
}

\section{Sven De Rijcke ${ }^{1}$, D. Michielsen ${ }^{2}$, P. Buyle ${ }^{1}$, D. J. Pisano ${ }^{3}$, H. Dejonghe ${ }^{1}$, and K. Freeman ${ }^{4}$}

${ }^{1}$ Sterrenkundig Observatorium, Universiteit Gent, Krijgslaan 281, S9, B-9000 Ghent, Belgium

${ }^{2}$ School of Physics and Astronomy, University of Nottingham, University Park, Nottingham NG7 2RD, UK

${ }^{3}$ Naval Research Laboratory, 4555 Overlook Avenue SW, Washington, DC 20375, USA

${ }^{4}$ Research School of Astronomy and Astrophysics, Australia National University, Cotter Road, ACT 2611, Australia

\begin{abstract}
We present deep Hi observations of a sample of 9 nearby E+A galaxies $(0.05<z<$ 0.1 ). In 7 of them, we detected up to a few times $10^{9} \mathrm{M}_{\odot}$ of neutral gas, making the link between $\mathrm{E}+$ As and early-types less direct than previously thought.
\end{abstract}

Keywords. galaxies: evolution, galaxies: ISM, radio lines: galaxies

Whereas clusters at $\mathrm{z} \sim 0.5$ contain a significant fraction of distorted blue galaxies, this population is almost missing in local clusters. About $60 \%$ of these blue galaxies, called E+A galaxies, are characterised by strong Balmer lines, typical for a very young stellar population, but have weak, if any, emission lines, suggesting the absence of star formation (Dressler et al. 1999). It has been suggested that E+As are witnessing the aftermath of a merger-triggered starburst and, over time, evolve into early-type galaxies (Poggianti et al. 1999). This would make E+As the crucial link between starbursting merger remnants and quiescent early-types, providing a direct observational window on the formation of massive galaxies.

We are conducting HI observations of a sample of $20 \mathrm{E}+\mathrm{A}$ galaxies (Buyle et al. 2006). Of the $9 \mathrm{E}+$ As observed so far, 7 have been detected at $21 \mathrm{~cm}$, containing significant amounts $\left(\sim 10^{9} M_{\odot}\right)$ of neutral hydrogen. These findings have important implications for the classification of E+As as post-starburst systems and as the progenitors of earlytype galaxies. With gas, the raw material for forming stars, still present, E+As may undergo future star-formation episodes, making the link between E+As and early-types less direct than previously thought. This also opens up the possibility that E+As are actively forming stars but that dust, which is surely present, obscures star-forming regions. Moreover, these results raise the question of why the starburst ended, if it did, before consuming all the gas. Possibly, the starburst evaporates the dense molecular clouds, temporarily halting star formation.

\section{References}

Buyle, P., Michielsen, D., De Rijcke, S., Pisano, D. J., Dejonghe, H., \& Freeman, K. 2006, ApJ, $649,163$.

Dressler, A., Smail, I., Poggianti, B. M., Butcher, H., Couch, W. J., Ellis, R. S., \& Oemler, A., Jr. 1999, ApJS, 122, 51.

Poggianti, B. M., Smail, I., Dressler, A., Couch, W. J., Barger, J., Butcher, H., Ellis, E. S., \& Oemler, A., Jr. 1999, ApJ, 518, 576. 